\title{
Sleep Related Eating Disorder as an Unexpected Effect of Zolpidem
}

\author{
Yuko Furuhashi* ${ }^{\circledR}$, Sumiko Satomura \\ Health Care Center, Shizuoka University, Shizuoka, Japan \\ Email: *furuhashi.yuko@shizuoka.ac.jp
}

How to cite this paper: Furuhashi, Y. and Satomura, S. (2019) Sleep Related Eating Disorder as an Unexpected Effect of Zolpidem. Neuroscience \& Medicine, 10, 75-81. https://doi.org/10.4236/nm.2019.102005

Received: March 15, 2019

Accepted: May 3, 2019

Published: May 6, 2019

Copyright $\odot 2019$ by author(s) and Scientific Research Publishing Inc. This work is licensed under the Creative Commons Attribution International License (CC BY 4.0).

http://creativecommons.org/licenses/by/4.0/

\begin{abstract}
Zolpidem is a sedative-hypnotic drug used to treat in sleep disorders, and it is the most commonly prescribed drug for insomnia. It reduces sleep latency and increases total sleep time. However, some studies have reported that zolpidem might induce sleep related eating disorder (SRED). SRED is characterized by recurrent episodes of compulsive and involuntary eating during night sleep, accompanied by partial consciousness and limited subsequent recall. The pathophysiology of SRED is unknown. Patients with SRED usually suffer from other sleep disorders such as sleepwalking, restless legs syndrome, and obstructive sleep apnea. In this article, we present an overview of case reports on SRED induced by zolpidem.
\end{abstract}

\section{Keywords}

Zolpidem, Sleep Related Eating Disorder, Insomnia

\section{Introduction}

Insomnia is a very common condition worldwide. Many patients with insomnia have only transient difficulties in falling asleep or staying awake, which resolve in a few days. However, approximately $20 \%$ of insomnia patients suffer from chronic insomnia [1]. Chronic insomnia impairs quality of life and reduces the ability to perform daily activities. Short-term treatments with benzodiazepine or non-benzodiazepine hypnotics are used to treat chronic insomnia [2]. Zolpidem ${ }^{1}$ is a non-benzodiazepine receptor agonist, and a highly effective hypnotic with a short half-life [3]. Compared with other hypnotics, zolpidem induces a pattern of sleep that is more similar to natural sleep. Several clinical trials that have evaluated zolpidem in the treatment of chronic insomnia have shown that the med-

${ }^{1}$ Zolpidem induced SRED. 
iation is well tolerated and remains effective for up to 12 months without any evidence of significant tolerance or rebound insomnia on discontinuation [3] [4]. In addition, zolpidem appears to have low abuse liability at doses commonly used to treat insomnia. Therefore, owing to these several clinical advantages, zolpidem is widely prescribed for treating insomnia.

However, some studies have reported that zolpidem is associated with sleep-related eating disorder (SRED) as well as sleepwalking [4]. According to the International Classification of Sleep Disorders, Second Edition [5], SRED is classified as parasomnia and is characterized by recurrent episodes of involuntary eating and drinking in the middle of sleep periods with problematic consequences. Patients have reported involuntary eating and their cohabitants find them to have difficulty in remaining to fully awake. This behavior may lead to morning bloating, social embarrassment, and weight gain. SRED is also associated with sleep disturbance, which can cause fatigue, somnolence, poor cognitive performance, irritability, and obesity [6]. The exact etiology of SRED is not clear [7]. It is often associated with other sleep disorders such as restless legs syndrome (RLS) and obstructive sleep apnea (OSA) [8]. In addition, SRED has been reported to be associated with and triggered by the use of psychotropic medications such as tricyclic antidepressants anticholinergics, olanzapine, and risperidone [9]. Recently, SRED has been implicated with the use of zolpidem [10]. The chemical structure of zolpidem is different from those of benzodiazepines, barbiturates, and other hypnotic drugs, and hence may be related to the development of SRED. Sporadic cases of zolpidem-induced SRED have been reported in the literature, and we recently experienced a case of zolpidem-induced SRED in a patient with attention-deficit hyperactivity disorder (unpublished data).

Therefore, we aimed to provide an overview of patients with SRED induced by zolpidem.

\section{Methods}

Electronic databases were searched through the academic search engine, MEDLINE. The following headings and keywords were used: 1) "zolpidem", 2) "sleep related eating disorder" or "SRED", and 3) "case" or "case report". The search sets included all papers published from January 2000 to January 2019. This search was limited to studies written in English. The search produced 19 articles. All the papers were then manually reviewed to ensure that the studies investigated SRED induced by zolpidem. Review articles were excluded. A total of 10 studies met the criteria for inclusion to the present study. Figure 1 shows the initial identification of 19 studies and the process through which the final 10 studies were selected for inclusion.

\section{Result}

\subsection{Overview of the Reviewed Studies}

A total of 10 case reports met the criteria for inclusion to this review. Table 1 provides 


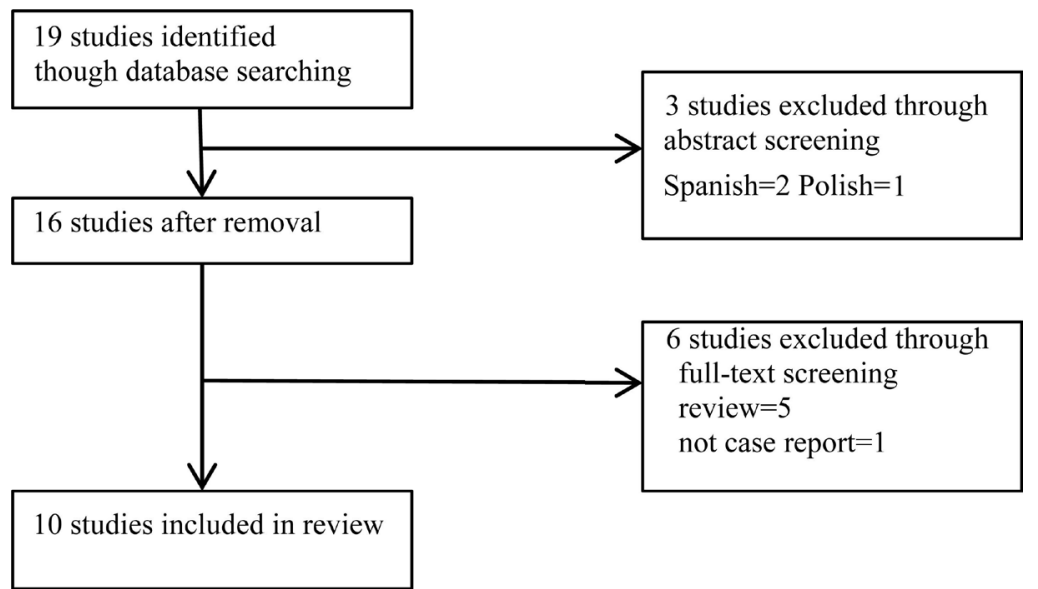

Figure 1. Flow chart for identifying studies.

Table 1. Summary of reviewed case reports.

\begin{tabular}{|c|c|c|c|c|c|}
\hline Author & $\begin{array}{c}\text { Age of } \\
\text { onset }\end{array}$ & Gender & $\begin{array}{c}\text { Recall of } \\
\text { nocturnal eating }\end{array}$ & $\begin{array}{l}\text { Zolpidem } \\
\text { (mg) }\end{array}$ & $\begin{array}{c}\text { Associated } \\
\text { sleep disorders }\end{array}$ \\
\hline Park Y.M. et al. [8] & 71 & male & amnestic & 12.5 & OSA, RLS \\
\hline Kim H.K.E. et al. [19] & 57 & female & full recall & 10 & RLS \\
\hline Nzwalo et al. [18] & 53 & female & amnestic & 10 & none \\
\hline \multirow[t]{8}{*}{ Wing Y.K. et al. [17] } & 61 & female & NA & 10 & OSA \\
\hline & 64 & male & NA & 10 & OSA \\
\hline & 63 & male & NA & 10 & OSA \\
\hline & 54 & female & NA & 10 & none \\
\hline & 56 & female & NA & 10 & narcolepsy \\
\hline & 44 & female & NA & 10 & none \\
\hline & 29 & female & NA & 10 & OSA \\
\hline & 28 & male & amnesic & 10 & OSA \\
\hline Hoque R., Chesson A.L. [16] & 51 & female & amnestic & 10 & OSA \\
\hline Yun C.H., Ji K.H. [15] & 45 & male & amnestic & 10 & RLS \\
\hline Dang A. et al. [14] & 45 & male & amnestic & 10 & none \\
\hline \multirow[t]{2}{*}{ Chiang A., Krystal A. [13] } & 75 & female & amnestic & $10-12.5$ & OSA \\
\hline & 70 & female & amnestic & $10-12.5$ & RLS, mild OSA \\
\hline Najjar M. et al. [12] & 46 & female & $\begin{array}{l}\text { amnestic - } \\
\text { partial recall }\end{array}$ & 6.25 & mild OSA \\
\hline \multirow[t]{5}{*}{ Morgenthaier T.I., Silber M.H. [11] } & 51 & female & amnestic & 10 & RLS \\
\hline & 65 & male & $\begin{array}{l}\text { some recall - } \\
\text { amnestic }\end{array}$ & $10-20$ & $\begin{array}{l}\text { RLS, OSA, } \\
\text { sleep walking }\end{array}$ \\
\hline & 41 & male & amnestic & $15-30$ & RLS, OSA \\
\hline & 64 & male & full recall & $5-10$ & RLS, OSA \\
\hline & 56 & female & amnestic & 10 & $\begin{array}{l}\text { RLS, sleep } \\
\text { walking }\end{array}$ \\
\hline
\end{tabular}

NA: Information not available; OSA: obstructive sleep apnea; RLS: restless leg syndrome. 
an overview of the reviewed articles. We found 22 cases of zolpidem-induced SRED. The average age of the patients was 54.0 years old (range 28 years to 71 years). Thirteen patients were female, 59.1\%; $13 / 22$ of cases. Zolpidem was prescribed for insomnia in all cases. In 22 cases, there were 13 (59.1\%) subjects were OSA, 9 (41.0\%) subjects were RLS, and 2 (9.1\%) subjects were sleep walking. Discontinuation of zolpidem led to remission of SRED in all cases.

\subsection{Details of Each Case}

Morgenthaler et al. [11] reported five cases (two females) of zolpidem induced SRED who were middle aged and had RLS. Two of these five patients had intermittent episodes of conscious nocturnal eating. Each patient began amnestic night eating, which stopped with discontinuation of zolpidem.

Najjar [12] reported the case of a 46-year-old woman who developed SRED after 3 weeks of taking $6.25 \mathrm{mg}$ of zolpidem. The patient had total amnesia or partial recall of night eating. After zolpidem was discontinued, nocturnal eating stopped completely.

Chiang et al. [13] reported two cases. The first was a 75-year-old woman with RLS and mild OSA. As soon as the patient was switched from zolpidem $10 \mathrm{mg}$ to $12.5 \mathrm{mg}$ of extended-release (ER) zolpidem, she developed amnestic SRED. After she stopped taking the ER zolpidem and resumed her usual zolpidem $10 \mathrm{mg}$ therapy, her SRED symptom resolved. The second case was a 70-year-old woman with RLS and sleep apnea. Soon after she was switched from zolpidem $10 \mathrm{mg}$ to $12.5 \mathrm{mg}$ of extended-release (ER) zolpidem, she developed amnestic SRED. After switching back to the immediate-release formulation of zolpidem, her nocturnal SRED resolved.

Dang et al. [14] reported the case of a 45-year-old man who was prescribed zolpidem and exhibited unexpected and bizarre eating behaviors during the somnambulistic state. After zolpidem was stopped, his SRED episodes were resolved.

Yun et al. [15] reported the case of a 45-year-old man with RLS and zolpidem-induced SRED. After zolpidem discontinuation, SRED did not recur. His RLS and related insomnia were controlled with pramipexole $0.5 \mathrm{mg}$ and clonazepam $0.25 \mathrm{mg}$.

Hoque et al. [16] reported the case of a 51-year-old woman with zolpidem-induced SRED. A few weeks after starting zolpidem $10 \mathrm{mg}$, she started sleep walking and eating. She also experienced one episode of sleep driving. After zolpidem was stopped, all sleep-related symptoms immediately ceased and did not recur.

Wing et al. [17] reported the cases of eight patients (five females) who presented with SRED while taking $10 \mathrm{mg}$ of zolpidem. The patients were reported to consume carbohydrates after a period of sleep. Six patients had comorbid OSA, for which one patient was already receiving continuous positive airway pressure (CPAP) treatment before the onset of SRED. None of the patients were diag- 
nosed with RLS or periodic limb movement disorder.

Nzmalo et al. [18] reported the case of a 53-year-old woman with zolpidem-induced SRED. Her family witnessed several episodes of sleep eating, trying to drink various types of food, and sometimes cook during sleep. During these episodes, her eyes remained almost closed. However, she was unable to remember anything related to the episodes, and it was suggested to video record one of the episodes. Subsequently, zolpidem was stopped and the night eating episodes ceased.

Kim et al. [19] reported the case of a 57-year-old woman with RLS. She had been receiving zolpidem $10 \mathrm{mg}$ daily for 6 months. During the treatment period, she ate forcefully without feeling hungry. Whenever her family members intervened during the eating episodes, she became angry and irritated. However, she could not recall her actions. Compulsive eating behavior disappeared after zolpidem administration was stopped.

Park et al. [8] reported the case of a 71-year-old man with SRED and complex behavior with anterograde amnesia. He had received $12.5 \mathrm{mg}$ of controlled-release zolpidem. He also suffered from RLS and OSA. His SRED disappeared immediately after zolpidem discontinuation.

\section{Discussion}

In this article, we present 22 cases of zolpidem-induced SRED. SRED is characterized by recurrent episodes of involuntary eating and drinking during arousals from sleep with problematic consequences. However, there was no study that investigated the prevalence of zolpidem-induced SRED. Moreover, the mechanism underlying the association between zolpidem and SRED remain unclear. It has been suggested that diffuse cortical binding of zolpidem might trigger central pattern generators causing emergence of conserved primitive patterns such as walking and eating. These primitive patterns could indeed be potentiated by the sedation-amnestic properties of zolpidem [16]. Some possible risk factors have been hypothesized, including zolpidem dosage, sex, drug-drug interaction with serotonin reuptake inhibitors, protein-binding competition, body weight, and a combination of past history of sleepwalking, medication increasing delta sleep, and a precipitating stimulus. Polypharmacy with concurrent medication was another risk for SRED. The uniqueness of zolpidem compared to other benzodiazepine and nonbenzodiazepine hypnotics with regard to precipitating SRED remains unclear. Further studies related to the pathophysiology of SRED are needed in the future. Zolpidem-induced SRED can usually be improved by discontinuing zolpidem.

To confirm a definite association between zolpidem and SRED, resolution of SRED on stopping zolpidem should be shown. Given that differences as small as formulation could affect the likelihood of SRED, it is practical for clinicians to keep in mind that SRED may be observed with the use of particular agents or at particular dosages in some subjects. Future research will be required to deter- 
mine whether it is possible to predict the pharmacologic profile that increases the risks of SRED related to zolpidem treatment 4.1.

\section{Conclusion}

Zolpidem is an effective hypnotic with a shorter half-life than other benzodiazepines. Zolpidem is widely prescribed owing to its favorable pharmacological properties for insomnia treatment. Clinicians should be aware of the unwarranted effects of zolpidem, provide appropriate management, and warn patients about the side effects of zolpidem use. Caution should be exercised while prescribing zolpidem to patients with insomnia. Future studies will be needed to reveal the relation between zolpidem and SRED.

\section{Conflicts of Interest}

The authors declare no conflicts of interest regarding the publication of this paper.

\section{References}

[1] Mprin, C.M., LeBlanc, M., Daley, M., Gregoire, J.P. and Merette, C. (2006) Epidemiology of Insomnia: Prevalence, Self-Help Treatment, Consultation, and Determinants of Help-Seeking Behaviors. Sleep Medicine, 7, 123-130. https://doi.org/10.1016/j.sleep.2005.08.008

[2] Ten Have, M., Penninx, B.W., van Dorsselaer, S., Tuithof, M., Kleinjan, M. and de Graaf, R. (2016) Insomnia among Current and Remitted Common Mental Disorders and the Association with Role Functioning: Results from a General Population Study. Sleep Medicine, 25, 34-41. https://doi.org/10.1016/j.sleep.2016.07.015

[3] Holm, K.J. and Goa, K.L. (2008) Zolpidem: An Update of Its Pharmacology, Therapeutic Efficacy and Tolerability in the Treatment of Insomnia. Drugs, 59, 865-889. https://doi.org/10.2165/00003495-200059040-00014

[4] Bomalaski, M.N., Claflin, E.S., Townsend, W. and Peterson, M.D. (2017) Zolpidem for the Treatment of Neurologic Disorders: A Systematic Review. JAMA Neurology, 74, 1130-1139. https://doi.org/10.1001/jamaneurol.2017.1133

[5] American Academy of Sleep Medicine (2005) The International Classification of Sleep Disorders: Diagnostic and Coding Manual. 2nd Edition, American Academy of Sleep Medicine, Westchester.

[6] Howell, M.J. and Schenck, C.H. (2009) Treatment of Nocturnal Eating Disorders. Current Treatment Options in Neurology, 11, 333-339.

https://doi.org/10.1007/s11940-009-0037-1

[7] Hwang, T.J., Ni, H.C., Chen, H.C., Lin, Y.T. and Liao, S.C. (2010) Risk Predictors for Hypnosedative-Related Complex Sleep Behaviors: A Retrospective, Cross-Sectional Pilot Study. Journal of Clinical Psychiatry, 71, 1331-1335. https://doi.org/10.4088/JCP.09m05083bro

[8] Park, Y.M. and Shin, H.W. (2016) Zolpidem Induced Sleep-Related Eating and Complex Behaviors in a Patient with Obstructive Sleep Apnea and Restless Legs Syndrome. Clinical Psychopharmacology and Neuroscience, 14, 299-301. https://doi.org/10.9758/cpn.2016.14.3.299

[9] Santin, J., Mery, V., Elso, M.J., Retamal, E., Torres, C., Ivelic, J. and Godoy, J. (2014) 
Sleep-Related Eating Disorder: A Descriptive Study in Chilean Patients. Sleep Medicine, 15, 163-167. https://doi.org/10.1016/j.sleep.2013.10.010

[10] Lam, S.P., Fong, S.Y., Ho, C.K., Yu, M.W. and Wing, Y.K. (2008) Parasomnia among Psychiatric Outpatients: A Clinical, Epidemiologic, Cross-Sectional Study. Journal of Clinical Psychiatry, 69, 1374-1382. https://doi.org/10.4088/JCP.v69n0904

[11] Morgenthaler, T.I. and Silber, M.H. (2002) Amnestic Sleep-Related Eating Disorder Associated with Zolpidem. Sleep Medicine, 3, 323-327. https://doi.org/10.1016/S1389-9457(02)00007-2

[12] Najjar, M. (2007) Zolpidem and Amnestic Sleep Related Eating Disorder. Journal of Clinical Sleep Medicine, 3, 637-638.

[13] Chiang, A. and Krystal, A. (2008) Report of Two Cases Where Sleep Related Eating Behavior Occurred with the Extended-Release Formulation But Not the Immediate-Release Formulation of a Sedative-Hypnotic Agent. Journal of Clinical Sleep Medicine, 4, 155-156.

[14] Dang, A., Garg, G. and Rataboli, P.V. (2009) Zolpidem Induced Noctrnal Sleep-Related Eating Disorder (NSRED) in a Male Patient. International Journal of Eating Disorders, 42, 385-386. https://doi.org/10.1002/eat.20620

[15] Yun, C.H. and Ji, K.H. (2010) Zolpidem-Induced Sleep-Related Eating Disorder. Journal of the Neurological Sciences, 288, 200-201. https://doi.org/10.1016/j.jns.2009.09.026

[16] Hoque, R. and Chesson, A.L.Jr. (2009) Zolpidem-Induced Sleepwalking, Sleep Related Eating Disorder, and Sleep-Driving: Fluorine-18-Flourodeoxyglucose Positron Emission Tomography Analysis, and a Literature Review of Other Unexpected Clinical Effects of Zolpidem. Journal of Clinical Sleep Medicine, 5, 471-476.

[17] Wing, Y.K., Lam, S.P., Li, S.X., Zhang, J. and Yu, M.W. (2010) Sleep-Related Eating Disorder and Zolpidem: An Open Interventional Cohort Study. Journal of Clinical Psychiatry, 71, 653-656. https://doi.org/10.4088/JCP.09105623gry

[18] Nzwalo, H., Ferreira, L., Peralta, R. and Bentes, C. (2013) Sleep-Related Eating Disorder Secondary to Zolpidem. BMJ Case Reports, 2013, bcr2012008003. https://doi.org/10.1136/bcr-2012-008003

[19] Kim, H.K., Kwon, J.T., Baek, J., Park, D.S. and Yang, K.I. (2013) Zolpidem-Induced Compulsive Evening Eating Behavior. Clinical Neuropharmacology, 36, 173-174. https://doi.org/10.1097/WNF.0b013e31829dd448 